REVISTA DE LA

UNIÓN MATEMÁTICA ARGENTINA

Vol. 59, No. 1, 2018, Pages 99-121

Published online: August 18, 2017

\title{
A UNIFIED POINT OF VIEW ON BOUNDEDNESS OF RIESZ TYPE POTENTIALS
}

\author{
BIBIANA IAFFEI AND LILIANA NITTI
}

\begin{abstract}
We introduce a natural extension of the Riesz potentials to quasimetric measure spaces with an upper doubling measure. In particular, these operators are defined when the underlying space has components of differing dimensions. We study the behavior of the potential on classical and variable exponent Lebesgue spaces, obtaining necessary and sufficient conditions for its boundedness. The technique we use relies on a geometric property of the measure of the balls which holds both in the doubling and non-doubling situations, and allows us to present our results in a unified way.
\end{abstract}

\section{INTRODUCTION}

The basic potential operators defined on real valued functions on $\mathbb{R}^{n}$ are the Riesz potentials or fractional integral operators $I_{\alpha}, 0<\alpha<n$, defined by

$$
I_{\alpha} f(x)=\int_{\mathbb{R}^{n}} \frac{f(y)}{|x-y|^{n-\alpha}} \mathrm{d} y,
$$

a definition which depends crucially on the dimension of the underlying Euclidean space.

If the base space is a fractal subset of $\mathbb{R}^{n}$, that is, a measurable set with noninteger Hausdorff dimension, the Riesz potentials of order $\alpha$ on the so-called $s$-sets are given by

$$
I_{\alpha}^{s} f(x)=\int_{\mathbb{R}^{n}} \frac{f(y)}{|x-y|^{s-\alpha}} \mathrm{d} \mu(y),
$$

where the role of the dimension is played by $s$. These Riesz potentials are considered in [43, 44, 39, where they are introduced as traces of the corresponding Euclidean variants.

When the underlying space $X$ has variable dimension $n(x), x \in X$, an appropriate Riesz type operator-reflecting the variability of the dimensions - may be defined by

$$
I_{\alpha}^{n(\cdot)} f(x)=\int_{X} \frac{f(y)}{|x-y|^{n(x)-\alpha}} \mathrm{d} \mu(y)
$$

2010 Mathematics Subject Classification. 47B38, $42 \mathrm{~B} 35$.

The authors were supported in part by CONICET, CAI+D (UNL) and ANCPyT. 
for a suitable measure $\mu$. A natural setting where this operator may arise is when the space $X$ consists of two components of differing dimensions, for instance, a plate joined to a block, or a rod joined to a plate. If the contact between the components is of order zero, we can use the doubling measure $\mu^{\gamma_{1}, \gamma_{2}}$ considered in [3], which - as we will show in Section 5.1 satisfies

$$
\mu^{\gamma_{1}, \gamma_{2}}(B(x, r)) \leq C r^{n(x)},
$$

for some constant $C$. Thus, $\mu^{\gamma_{1}, \gamma_{2}}$ is an example of an upper doubling measure with dominating function $\mathrm{Cr}^{n(x)}$, which reflects the changing dimensions.

All this suggests that we study the following more general operator which extends that in 1.2 :

$$
I_{\alpha}^{\lambda} f(x)=\int_{X} \frac{d(x, y)^{\alpha}}{\lambda(x, d(x, y))} f(y) \mathrm{d} \mu(y),
$$

where $(X, d, \mu)$ is a quasi-metric space with upper doubling measure associated with the dominating function $\lambda$, i.e., $\mu(B(x, r)) \leq \lambda(x, r)$. The so-called upper doubling measures, introduced in [25], constitute a simultaneous generalization of doubling, upper Ahlfors $n$-regular and variable upper Ahlfors $n(\cdot)$-regular measures.

The classical case of $I_{\alpha}$ is recovered from 1.4 when $X=\mathbb{R}^{n}, d$ is the usual Euclidean metric on $\mathbb{R}^{n}, \mu$ is the Lebesgue measure on $\mathbb{R}^{n}$, and $\lambda(x, r)=C r^{n}$. The boundedness of these operators was studied by Hardy and Littlewood [18, 19] and Sobolev [36]. They proved that $I_{\alpha}$ maps a function in $L^{p}$ boundedly to a function in $L^{q}$, provided that $\frac{1}{q}=\frac{1}{p}-\frac{\alpha}{n}$. This result was proved also using interpolation in [37, and employing a pointwise estimation involving maximal functions in 22.

Norm estimates for $I_{\alpha} f$ in Lebesgue spaces, as well as for operators with more general kernels than $|x-y|^{\alpha-n}$ and defined on more general quasi-metric spaces, have been extensively studied in the doubling and non-doubling contexts. For instance, in [13] and [29] we find:

Theorem 1.1. Let $(X, d, \mu)$ be a quasi-metric measure space and let $0<\alpha<n$, $1<p<\frac{n}{\alpha}$ and $\frac{1}{q}=\frac{1}{p}-\frac{\alpha}{n}$. Then

$$
I_{\alpha}: L^{p}(X, d, \mu) \rightarrow L^{q}(X, d, \mu)
$$

boundedly if and only if

$$
\mu(B(x, r)) \leq c_{1} r^{n}
$$

where the constant $c_{1}$ is independent of $x$ and $r$.

Let us state now the main result of this paper, which allows us to derive the above statement as an immediate consequence. The precise definitions and properties stated below will be given in Section 2

Theorem 1.2. Let us assume that $(X, d)$ is a bounded geometrically doubling quasimetric space and $\mu$ is a Borel measure on $X$ defined on d-balls, which is finite on bounded sets and positive on d-balls. Let $\lambda: X \times \mathbb{R}^{+} \rightarrow \mathbb{R}^{+}$be a function, which as a function of the variable $r$ and uniformly on $x$ is non-decreasing, doubling, of lower type greater than $\alpha$ with $\alpha>0$. Let $q$ be a measurable function defined on $X$, 
such that $1<p<q_{-} \leq q(x) \leq q_{+}<\infty$ and $q(x)$ satisfies $r^{\alpha}=\lambda(x, r)^{\frac{1}{p}-\frac{1}{q(x)}}$ for all $x \in X$ and $r>0$. Then,

$$
I_{\alpha}^{\lambda}: L^{p}(X, d, \mu) \rightarrow L^{q(\cdot)}(X, d, \mu)
$$

boundedly if and only if

$$
\mu(B(x, r)) \leq \lambda(x, r)
$$

for all $x \in X$ and $0<r \leq \operatorname{diam}(X)$.

We observe that this theorem solves the boundedness problem for the operator defined by 1.2 in Lebesgue spaces on $(X,|\cdot|, \mu)$ where $\mu$ satisfies $\mu(B(x,|x-y|)) \leq$ $|x-y|^{n(x)}$ for every $x \in X$. Our proof of the sufficient condition for boundedness follows an idea from [29], and allows us to unify the proofs for the homogeneous and non-homogeneous cases. The basic strategy is to consider an adequate maximal function involving the measure of the balls and the dominating function $\lambda$.

Our work also extends the Riesz potential to the more general situation where there may be many (non-overlapping) fractals embedded in $\mathbb{R}^{n}$, eventually with different Hausdorff dimensions and which may or may not touch each other. In this variable dimension context, we must mention the work by Hambly and Kumagai [17, who studied diffusion processes on fractal components embedded in $\mathbb{R}^{2}$. Physical examples related to this subject may be found in [5, 10, 38,

The variable dimension case was considered in [20, where it was proved the boundedness in variable Lebesgue spaces of the operator

$$
I_{\alpha} f(x)=\int_{X} \frac{d(x, y)^{\alpha}}{\mu(B(x, d(x, y)))} f(y) \mathrm{d} \mu(y),
$$

by assuming that the measure is lower Ahlfors $n(\cdot)$-regular in a bounded subset $X$ of $\mathbb{R}^{n}$.

We note that our definition in $\sqrt{1.2}$ is different from that in 1.5 , as it carries information on the behavior of the dimension. In this paper, unlike the result in [20, we obtain the boundedness on Lebesgue spaces of $I_{\alpha}^{n(\cdot)}$ by requiring $(X, d)$ to be a bounded geometrically doubling quasi-metric space and $\mu$ to be upper Ahlfors $n(\cdot)$-regular. More precisely, if $\frac{1}{q(x)}=\frac{1}{p}-\frac{\alpha}{n(x)}$ then the operator $I_{\alpha}^{n(\cdot)}$ is a bounded operator from $L^{p}(X, d, \mu)$ into $L^{q(\cdot)}(X, d, \mu)$ with $\mu(B(x, r)) \leq C r^{n(x)}$. The boundedness of $I_{\alpha}^{n(\cdot)}$ remains valid for variable exponent Lebesgue spaces $L^{p(\cdot)}$ if the exponent $p$ is $\log$-Hölder continuous and $\frac{1}{q(x)}=\frac{1}{p(x)}-\frac{\alpha}{n(x)}$.

Sufficient conditions for the boundedness in Lebesgue spaces of the operator defined by

$$
I_{\alpha} f(x)=\int_{X} \frac{1}{(\lambda(y, d(x, y)))^{1-\alpha}} f(y) \mathrm{d} \mu(y),
$$

when $\mu$ is an upper doubling measure with dominating function $\lambda$, are studied in [11] as a particular case of the operators $T_{\alpha} f(x)=\int_{X} K_{\alpha}(x, y) f(y) \mathrm{d} \mu(y)$, where the kernel $K_{\alpha}$ satisfies $K_{\alpha}(x, y) \leq C_{K_{\alpha}} \frac{1}{(\lambda(y, d(x, y)))^{1-\alpha}}$. In our case, $K_{\alpha}(x, y)=$ $\frac{d(x, y)^{\alpha}}{d(x, y)^{n(x)}}$ and $\lambda(x, d(x, y))=d(x, y)^{n(x)}$, so the condition on $K_{\alpha}$ required in [1] 
is only satisfied if $n(x)<1$. Therefore, this result cannot be applied to study the boundedness in Lebesgue spaces of the operator in $(1.2)$.

In Section 2 we describe our setting in more detail, state the definitions of the basic concepts, and present a condition under which the upper doubling measure cannot have atoms, obtaining as a consequence that the underlying space has no isolated points if the balls have positive measure.

Section 3 is devoted to introducing the appropriate Riesz potential operator $I_{\alpha}^{\lambda}$ in an upper doubling environment, and to showing that the latter encompasses the doubling and non-doubling cases. In Section 4 we give necessary and sufficient conditions on the measure for which the boundedness for $I_{\alpha}^{\lambda}$ on Lebesgue spaces holds. In Subsection 5.1 we study regularity properties of a measure defined in [3], and show that this measure is another non-trivial example of an upper doubling measure. In Subsection 5.2 we state the sufficient condition for the boundedness of $I_{\alpha}^{n(\cdot)}$ on Lebesgue spaces.

\section{The GENERAL SETTING AND BASIC FACTS}

2.1. Quasi-metric measure spaces. Let $(X, d)$ a quasi-metric space. By a quasimetric on a set $X$ we mean a nonnegative function $d$ defined on $X \times X$ such that

- $d(x, y) \geq 0$ for every $x$ and $y$ in $X$ and $d(x, y)=0$ if and only if $x=y$;

- $d(x, y)=d(y, x)$ for every $x$ and $y$ in $X$;

- $d(x, y) \leq K_{1}(d(x, z)+d(z, y))$ for every $x$ and $y$ in $X$ and for some finite constant $K_{1}>0$.

A quasi-metric space $(X, d)$ is geometrically doubling or has the weak homogeneity property if there exists a natural number $N$ such that every $d$-ball $B(x, r)=$ $\{y \in X: d(y, x)<r\}$ can be covered by at most $N d$-balls of radius $r / 2$. A basic observation is that in a geometrically doubling quasi-metric space, a $d$-ball $B(x, r)$ can contain the centers $x_{i}$ of at most $N \alpha^{-n}$ disjoint $d$-balls $B\left(x_{i}, \alpha r\right)$ for $\alpha \in(0,1]$. This weak homogeneity was first observed by Coifman and Weiss in [7]. Hytönen in [25] gives other equivalent conditions of that definition. As it was shown by Macías and Segovia in 32, every quasi-metric space is metrizable in the sense that there exist a distance $\rho$ and a positive number $\alpha$ such that $\rho^{\alpha}$ is equivalent to $d$. The geometrically doubling quasi-metric spaces also satisfy the following topological properties: they are separable and have the Heine-Borel property (see [1]).

A Borel measure $\mu$ defined on the $d$-balls is said to be non-trivial if $\mu(B(x, r))$ is positive and finite for every $x \in X$ and every $r>0$. A non-trivial measure $\mu$ is said to be doubling if there exists a positive constant $K_{2}$ such that the inequality

$$
\mu(B(x, 2 r)) \leq K_{2} \mu(B(x, r))
$$

holds for every $x \in X$ and every $r>0$. We say that $(X, d, \mu)$ is a space of homogeneous type if $\mu$ is doubling on $(X, d)$. There is an extensive literature on analysis on these structures, in particular several examples and applications are given in [7].

It is well known that if $(X, d)$ supports a doubling measure then $(X, d)$ is geometrically doubling. Indeed, it was one of the first things pointed out by Coifman 
and Weiss in [7, p. 67]. Luukkainen and Saksman [31 proved that if $(X, d)$ is a complete, geometrically doubling metric space, then there exists a Borel measure $\mu$ on $X$ such that $(X, d, \mu)$ is a space of homogeneous type. Also, a compact metric space carries a non-trivial doubling measure if and only if it is a geometrically doubling metric space [40, 42].

We say that a point $x$ in a space of homogeneous type $(X, d, \mu)$ is an atom if $\mu(\{x\})>0$. When $\mu(\{x\})=0$ for every $x \in X$ we say that $(X, d, \mu)$ is a non-atomic space. In [32], Macías and Segovia proved in the context of spaces of homogeneous type, that a point is an atom if and only if it is topologically isolated, and that the set of such points is at most countable.

In this article we assume that $\operatorname{diam}(X)<\infty$. Then there exists a positive constant $R_{0}=\operatorname{diam}(X)$ such that

$$
X=B\left(x, R_{0}\right)
$$

for all $x \in X$.

As it is known, from (2.1) the following property holds

$$
\frac{\mu(B(x, \rho))}{\mu(B(y, r))} \geq C_{\mu}\left(\frac{\rho}{r}\right)^{N}, \quad N=\log _{2} K_{2},
$$

for all the balls $B(x, \rho)$ and $B(y, r)$ with $0<r \leq \rho<\infty$ and $y \in B(x, \rho)$, where $C_{\mu}>0$ does not depend on $r, \rho$ and $x$. From 2.3 we have

$$
\mu(B(x, r)) \geq c_{0} r^{N}, \quad x \in X, 0<r \leq \operatorname{diam}(X) .
$$

Condition (2.4) is also known as the lower Ahlfors regularity condition. The upper Ahlfors regularity condition (also called the non-doubling condition) holds on $X$ if there exists $n>0$ such that

$$
\mu(B(x, r)) \leq c_{1} r^{n},
$$

where $c_{1}>0$ does not depend on $x \in X$ and $0<r \leq \operatorname{diam}(X)$, and $n$ need not be an integer. It is noteworthy that power bounded measures are just different, not more general, than the doubling measures.

Given a Borel measure $\mu$ on $X$, we say that $(X, d, \mu)$ is an Ahlfors $Q$-regular quasi-metric measure space or a $Q$-normal space, for $Q>0$, if there exists a constant $A_{1} \geq 1$, such that

$$
A_{1}^{-1} r^{Q} \leq \mu(B(x, r)) \leq A_{1} r^{Q},
$$

for $0<r \leq \operatorname{diam}(X)$ and $x \in X$. It is easy to show that if $(X, d, \mu)$ is an Ahlfors $Q$-regular quasi-metric measure space, then the Hausdorff dimension, with respect to $d$, is exactly $Q$. Moreover for $Q>0$ no upper Ahlfors $Q$-regular quasi-metric measure space has atoms, in the sense that no single point has positive $\mu$ measure. In particular, if $\mu$ is positive on all $d$-balls and it satisfies the upper Ahlfors $Q$ regular condition, the space has not isolated points. Likewise, no lower Ahlfors $Q$-regular quasi-metric measure space has isolated points.

If in the above definition the variation of $r$ is modified as $\frac{\mu(\{x\})}{A_{1}}<r \leq \operatorname{diam}(X)$, the case of bounded spaces with atoms is also taken into account. When $Q=1$ 
the space $(X, d, \mu)$ is called normal space (see [32]), while when $Q \neq 1$ it is usually called $Q$-normal.

It is also of interest to study Ahlfors $Q(\cdot)$-regular spaces with a variable dimension. Along this line, if $Q: X \rightarrow(0, \infty)$ is a bounded function, we say that $\mu$ is Ahlfors $Q(\cdot)$-regular when $\mu(B(x, r)) \approx r^{Q(x)}$, for all $x \in X$ and $0<r \leq \operatorname{diam} X$. Ahlfors $Q(\cdot)$-regularity is possible only for sufficiently regular functions $Q$ (see [20]). Similarly, a measure $\mu$ is lower Ahlfors $N(\cdot)$-regular if $\mu(B(x ; r)) \geq c_{0}^{\prime} r^{N(x)}$ or upper Ahlfors $n(\cdot)$-regular if $\mu(B(x ; r)) \leq c_{1}^{\prime} r^{n(x)}$ for all $x \in X$ and $0<r \leq \operatorname{diam}(X)$.

In [25], Hytönen defines a class of measures which are controlled from above by appropriate doubling functions, including in this way both, doubling measures as well as those satisfying $\mu(B(x ; r)) \leq c_{1} r^{n}$ or even more generally, $\mu(B(x ; r)) \leq$ $c_{1}^{\prime} r^{n(x)}$. More precisely, a Borel measure $\mu$ on some quasi-metric space $(X, d)$ defined on $d$-balls is called upper doubling if there exists a dominating function $\lambda: X \times \mathbb{R}^{+} \rightarrow \mathbb{R}^{+}$so that $r \rightarrow \lambda(x, r)$ is non-decreasing, doubling (there exists a constant $C_{\lambda}$ such that $\lambda(x, 2 r) \leq C_{\lambda} \lambda(x, r)$ for every $x \in X$ and $\left.r>0\right)$ and

$$
\mu(B(x, r)) \leq \lambda(x, r) \quad \text { for all } x \in X \text { and } r>0 .
$$

A quasi-metric measure space $(X, d, \mu)$ is said to be upper doubling if $\mu$ is an upper doubling measure. The number $\operatorname{dim}:=\log _{2} C_{\lambda}$ can be thought of as (an upper bound for) a dimension of the measure $\mu$, and it plays a similar role to the quantity denoted by $N$ in 2.3. It was proved in [27] that there always exists another dominating function $\lambda$ such that $\tilde{\lambda} \leq \lambda, C_{\tilde{\lambda}} \leq C_{\lambda}$ and, for all $x, y \in X$ with $d(x, y)<r$,

$$
\tilde{\lambda}(x, r) \leq C_{\tilde{\lambda}} \tilde{\lambda}(y, r) .
$$

Thus in what follows, we assume that $\lambda$ satisfies (2.7).

It is immediate that a doubling measure is a special case of upper doubling, where one can take the dominating function to be $\lambda(x, r)=\mu(B(x, r))$. On the other hand, a non-doubling measure is upper doubling with $\lambda(x, r)=C r^{n}$. In [26], Hytönen and Martikainen note that the measures obtained by Volberg and Wick in [41] are actually upper doubling. In Section 5.1 we show that the measure defined by $\mathrm{H}$. Aimar and one the authors in [3] is another non-trivial example of upper doubling measure and moreover is an upper Ahlfors $n(\cdot)$-regular measure.

In the following lemma we state a relation between upper doubling measures and atoms.

Lemma 2.1. If $\mu$ is an upper doubling measure on $X$ with a dominating function $\lambda$ satisfying for each $x \in X$ that $\lambda\left(x, r_{j}\right) \rightarrow 0$ when $r_{j} \rightarrow 0$ as $j \rightarrow \infty$, then $\mu$ has no atoms. If, additionally, the measure is positive on the balls, the space has no isolated points.

Proof. Suppose on the contrary that there exists $x \in X$ such that $\mu(\{x\})=\alpha>0$, with $\mu(\{x\}) \leq \mu\left(B\left(x, r_{j}\right)\right) \leq \lambda\left(x, r_{j}\right)$. Then, using the hypotheses, we obtain $\alpha \leq 0$, contradicting the assumption about $\alpha$. The proof of the second part is immediate since if $x$ is an isolated point then there is a positive number $R$ such that $\{x\}=B(x, R)$. 
Remark 2.2. Note that the above property on $\lambda$ is satisfied in the case $\lambda(x, r)=C r^{n}$ and when $\lambda(x, r)=\mu(B(x, r))$ it is translated as $\mu\left(B\left(x, r_{j}\right)\right) \rightarrow 0$ for each $x$ when $r_{j} \rightarrow 0$ for $j \rightarrow \infty$.

Remark 2.3. On the other hand, if $\lambda$ as function of the variable $r$ and uniformly on $x$ is of lower type $a$ (see Subsection 2.4 below), then $\lambda$ satisfies the inequality $\lambda(x, r) \leq c_{1} r^{a} \lambda(x, 1)$ for some constant $c_{1}, a>0$ and for $0<r<1$, and thus $\lambda$ is in the conditions of Lemma 2.1

We refer the reader to [1, 9, 14, 16, 23, for general properties of quasi-metric measure spaces.

2.2. The modified maximal operator. Let $(X, d)$ be a geometrically doubling quasi-metric space and $\mu$ be a Borel measure on $X$ which is finite on bounded sets. Recall that the Hardy-Littlewood maximal function $M f(x)$ is defined (for Borel measurable functions $f$ ) by

$$
M f(x):=\sup _{r>0} \frac{1}{\mu(B(x, r))} \int_{B(x, r)}|f| \mathrm{d} \mu .
$$

The definition makes sense $\mu$-almost everywhere since if $x$ belongs to the support of $\mu$, then $\mu(B(x, r))$ is positive for every $r>0$ (otherwise a small open ball centered at $x$ could be removed from the support of $\mu$ ). If the measure $\mu$ satisfies the doubling property, it is well known that the Hardy-Littlewood maximal operator is bounded on all $L^{p}(\mu)$ with $1<p \leq+\infty$ and from $L^{1}(\mu)$ into $L^{1, \infty}(\mu)$. However if we omit the doubling requirement of the measure, for an arbitrary geometrically doubling quasi-metric space $X$ and measure $\mu$, we can only say that $M$ is bounded on $L^{\infty}(\mu)$. One way to avoid this problem is to replace the measure of the $d$-ball $B(x, r)$ by the measure of an appropriate dilation of the ball. More precisely, we define

$$
\tilde{M} f(x):=\sup _{r>0} \frac{1}{\mu\left(B\left(x, 3 K_{1} r\right)\right)} \int_{B(x, r)}|f| \mathrm{d} \mu,
$$

where $K_{1}$ is the constant in the definition of a quasi-metric. Note that always $\tilde{M} f(x) \leq M f(x)$ and, if the measure $\mu$ satisfies the doubling condition, $\tilde{M} f(x) \leq$ $C M f(x)$ for some constant $C>0$.

Lemma 2.4. If $(X, d)$ is geometrically doubling, and $\mu$ is a Borel measure on $X$ which is finite on bounded sets, the modified maximal operator $\tilde{M}$ is bounded on $L^{p}(\mu)$ for each $p \in(1 ; \infty]$ and acts from $L^{1}(\mu)$ into $L^{1, \infty}(\mu)$.

The weak type 1-1 estimate has been proved by Nazarov, Treil and Volverg in [34]. For a different approach see [9], p. 368, and the references therein.

2.3. Variable exponent Lebesgue spaces. In this subsection we recall some basic definitions and properties of variable exponent Lebesgue spaces, that we will need in Sections 4 and 5.2

Let $p: X \rightarrow[1 ; \infty)$ be a $\mu$-measurable function. We define

$$
p_{-}=\operatorname{essinf}_{x \in X} p(x) \quad \text { and } \quad p_{+}=\underset{x \in X}{\operatorname{ess} \sup } p(x) .
$$


Everywhere below we assume that

$$
1<p_{-} \leq p(x) \leq p_{+}<\infty, \quad x \in X .
$$

By $L^{p(\cdot)}(X)$ we denote the space of all $\mu$ measurable functions $f$ on $X$ such that the modular

$$
I_{p(\cdot)}(f)=I_{p(\cdot) ; X}(f):=\int_{X}|f(x)|^{p(x)} \mathrm{d} \mu(x)
$$

is finite. This turns out to be a Banach space with respect to the norm

$$
\|f\|_{p(\cdot)}=\|f\|_{p(\cdot) ; X}:=\inf \left\{\lambda>0: I_{p(\cdot)}\left(\frac{f}{\lambda}\right) \leq 1\right\} .
$$

It can be seen in [30] that $I_{p(\cdot)}$ has the following properties:

(i) $I_{p(\cdot)}(f) \geq 0$ for every function $f$.

(ii) $I_{p(\cdot)}(f)=0$ if and only if $f=0$.

(iii) $I_{p(\cdot)}(-f)=I_{p(\cdot)}(f)$ for every $f$.

(iv) $I_{p(\cdot)}$ is convex.

(v) If $|f(x)| \geq|g(x)|$ for a.e. $x \in X$ and if $I_{p(\cdot)}(f)<\infty$, then $I_{p(\cdot)}(f) \geq I_{p(\cdot)}(g)$; the last inequality is strict if $|f| \neq|g|$. (vi)

$$
\text { If }\|f\|_{p(\cdot)} \leq 1, \text { then } I_{p(\cdot)}(f) \leq\|f\|_{p(\cdot)} .
$$

The properties (i)-(iv) characterize $I_{p(\cdot)}$ as the convex modular in the sense of [33].

In the setting of quasi-metric measure spaces $(X, d, \mu)$, a version of Theorem 2.8 in [30. can be proved, namely that $L^{q(x)}$ is continuously embedded in $L^{p(x)}$ if and only if $p(x) \leq q(x)$ for a.e. $x \in X$, when $0<\mu(X)<\infty$ and $p, q$ are measurable functions such that $p, q: X \rightarrow[1, \infty)$. This result will be used in Theorem 4.6

Variable exponent Lebesgue spaces on general quasi-metric measure spaces have been considered in [21, 20, 12, 4, 15].

2.4. Functions of lower and upper type. Let us recall some definitions concerning increasing functions which appear in the literature when generalizing power functions.

We say that an increasing function $\lambda:(0, \infty) \rightarrow(0, \infty)$ is of lower type $a \geq 0$ if

$$
\lambda(s t) \leq c_{1} s^{a} \lambda(t)
$$

for some constant $c_{1}$, every $0<s \leq 1$, and every $t>0$. Similarly $\lambda$ is of upper type $b \geq 0$ if

$$
\lambda(s t) \leq c_{2} s^{b} \lambda(t)
$$

for some constant $c_{2}$, every $s \geq 1$, and every $t>0$. It is immediate that if $\lambda$ is of lower type $a_{1}$ and $a_{2}<a_{1}$ then $\lambda$ is also of lower type $a_{2}$. We say that a function is of lower type greater than $\alpha$ if it is of lower type $\alpha_{0}$, for some $\alpha_{0}>\alpha$. Similarly for upper type less than $\alpha$.

Now we state a property that will be useful in the following sections. 
Proposition 2.5. Let $\lambda$ be an increasing function and let $\alpha$ be a positive real number. Then $\lambda$ is of lower type $\alpha$ if and only if the inequality

$$
\frac{r_{2}^{\alpha}}{\lambda\left(r_{2}\right)} \leq c_{1} \frac{r_{1}^{\alpha}}{\lambda\left(r_{1}\right)}
$$

holds for all $0<r_{1} \leq r_{2}$ and some positive constant $c_{1}$.

Proof. Suppose first that 2.11 holds and $0<s \leq 1$, so that $s t \leq t$ for all $t>0$. Then 2.11 takes the following form:

$$
\frac{t^{\alpha}}{\lambda(t)} \leq c_{1} \frac{(s t)^{\alpha}}{\lambda(s t)}
$$

From this we obtain that $\lambda$ is of lower type $\alpha$. Conversely, if $\lambda$ is of lower type $\alpha$ and $0<r_{1} \leq r_{2}$ then

$$
\lambda\left(r_{1}\right)=\lambda\left(\frac{r_{1}}{r_{2}} r_{2}\right) \leq c_{1}\left(\frac{r_{1}}{r_{2}}\right)^{\alpha} \lambda\left(r_{2}\right)
$$

and 2.11 is satisfied.

\section{The Riesz OPERATOR IN AN UPPER DOUBLING ENVIRONMENT}

As we mentioned, the fractional integral operator or the Riesz potential $I_{\alpha}$, $0<\alpha<n$, is defined by

$$
I_{\alpha} f(x)=\int_{\mathbb{R}^{n}} \frac{f(y)}{|x-y|^{n-\alpha}} \mathrm{d} y,
$$

$x \in \mathbb{R}^{n}$, whenever this integral is finite. Clearly, if $f$ is a bounded function with compact support, then the integral in 3.1 is finite for every $x$. This operator was first studied by Hardy and Littlewood in the 1920's [18, 19] and extended by Sobolev [36] in the 1930's. A well known result for $I_{\alpha}$ is the Hardy-LittlewoodSobolev inequality: $\left\|I_{\alpha} f\right\|_{L^{q}} \leq C_{p, q}\|f\|_{L^{p}}$, that is, it is bounded from $L^{p}\left(\mathbb{R}^{n}\right)$ into $L^{q}\left(\mathbb{R}^{n}\right)$ if and only if $\frac{1}{q}=\frac{1}{p}-\frac{\alpha}{n}$, with $1<p<\frac{n}{\alpha}$ (see, e.g., [37]).

These statements were generalized in many directions; see the book [35] for historical notes and review of results.

As we pointed out in the introduction, also fractional integrals over quasi-metric measure spaces have been defined in different ways. There are natural extensions to contexts of quasi-metric measure spaces that arise from considering $|x-y|^{n}=$ $\mu(B(x,|x-y|))$, with $\mu$ the $n$-dimensional Lebesgue measure, or simply by thinking of $|x-y|^{n}$ as the $n$-dimensional power of the Euclidean distance between $x$ and $y$, or even regarding $|x-y|^{n}$ as a quasi-distance between $x$ and $y$.

In what follows, we shall assume that $(X, d)$ is a geometrically doubling quasimetric space, the $d$-balls are open sets, and that we have a Borel measure $\mu$ on $X$ which is finite on bounded sets, positive on the $d$-balls, and $\mu(\{x\})=0$ for all $x \in X$. 
Let us consider the following operators of potential type:

$$
\begin{aligned}
I_{\alpha}^{Q} f(x) & =\int_{X} f(y) \frac{1}{d(x, y)^{Q-\alpha}} \mathrm{d} \mu(y), \quad 0<\alpha<Q . \\
I_{\gamma} f(x) & =\int_{X} f(y) \frac{1}{d(x, y)^{1-\gamma}} \mathrm{d} \mu(y), \quad 0<\gamma<1 . \\
K_{\gamma} f(x) & =\int_{X} f(y) \frac{1}{\mu(B(x, d(x, y)))^{1-\gamma}} \mathrm{d} \mu(y), \quad 0<\gamma<1 . \\
J_{\alpha} f(x) & =\int_{X} f(y) \frac{d(x, y)^{\alpha}}{\mu(B(x, d(x, y)))} \mathrm{d} \mu(y), \quad \alpha>0 .
\end{aligned}
$$

We observe that from the results obtained in [32, it turns out that, given an arbitrary space of homogeneous type $(X, d, \mu)$, there exists a normal space $(X, \delta, \mu)$ of order $\theta, \theta>0$, such that the $L^{p}(X, d, \mu)$ coincides with $L^{p}(X, \delta, \mu)$. Therefore, in the case of $\mu$ doubling, the study of the boundedness of $I_{\gamma}$ or $K_{\gamma}$ is indistinct, because both operators are equivalent.

Obviously, if $\mu$ is Ahlfors $Q$-regular, then $I_{\alpha}^{Q}$ and $J_{\alpha}$ are equivalent. This is what happens for example when we consider the case of $s$-sets and $\mu$ is the restriction of the Hausdorff $s$-measure $\mathcal{H}^{s}$ to those sets.

If $\mu$ is doubling, using 2.4 we have $J_{\alpha} f(x) \leq \frac{1}{c_{0}} I_{\alpha}^{N} f(x), f \geq 0$. Similarly, $I_{\alpha}^{n} f(x) \leq J_{\alpha} f(x), f \geq 0$, when $\mu$ is "non-doubling", i.e., 2.5 holds. Moreover it can be seen ([29, 13]) that for a measure $\mu$, finite over balls and not having any atoms, condition 2.5 is necessary for the inequality $\left\|I_{\alpha}^{n}\right\|_{q} \leq C\|f\|_{p}, \frac{1}{q}=\frac{1}{p}-\frac{\alpha}{n}$ to hold.

In the general case, $c_{0} r^{N} \leq \mu(B(x, r)) \leq c_{1} r^{n}$, where $n \leq N$ and $r \in(0,1)$, the operator $J_{\alpha}$ is better suited for lower Ahlfors $N$-regular quasi-metric measure spaces, while $I_{\alpha}^{n}$ adjusts better for upper Ahlfors $n$-regular quasi-metric measure spaces.

The four potential type integral operators defined above can be viewed as special cases of the operator

$$
I_{\alpha}^{\lambda} f(x)=\int_{X} \frac{d(x, y)^{\alpha}}{\lambda(x, d(x, y))} f(y) \mathrm{d} \mu(y),
$$

where $\lambda$ is a dominating function for the upper doubling measure $\mu$ and as function of the variable $r$ and uniformly on $x$ is of lower type greater than $\alpha$. Clearly, if $f$ is a bounded function supported on a set of finite measure, then the integral in 3.2 is finite for almost every $x \in X$, but according to 2.2 we have $X=B\left(x, R_{0}\right)$, so it will be enough to require $f$ bounded.

\section{Boundedness of $I_{\alpha}^{\lambda}$ In Lebesgue spaces}

Now we state the first half of our main result, which gives a version of the Hardy-Littlewood-Sobolev inequality in the context of upper doubling spaces. We describe those quasi-metric measure spaces on which the potential type operator maps $I_{\alpha}^{\lambda}: L^{p}(X, d, \mu) \rightarrow L^{q(\cdot)}(X, d, \mu)$ boundedly. 
Theorem 4.1 (Sufficient Condition). Let us assume that $(X, d)$ is a bounded geometrically doubling quasi-metric space and $\mu$ is a Borel measure on $X$ defined on $d$-balls which is finite on bounded sets and positive on d-balls. Let $\lambda: X \times \mathbb{R}^{+} \rightarrow \mathbb{R}^{+}$ be a function, which as a function of the variable $r$ and uniformly on $x$ is nondecreasing, doubling, of lower type greater than $\alpha$ with $\alpha>0$. Let $q$ be a measurable function defined on $X$, such that $1<p<q_{-} \leq q(x) \leq q_{+}<\infty$ and $q(x)$ satisfies $r^{\alpha} \leq \lambda(x, r)^{\frac{1}{p}-\frac{1}{q(x)}}$ for all $x \in X$ and $r>0$. Then,

$$
I_{\alpha}^{\lambda}: L^{p}(X, d, \mu) \rightarrow L^{q(\cdot)}(X, d, \mu)
$$

boundedly if

$$
\mu(B(x, r)) \leq \lambda(x, r)
$$

for all $x \in X$ and $0<r \leq \operatorname{diam}(X)$.

Proof. We are going to adapt to our context the proof given by Hedberg in [22].

Let $B=B(x, r), x \in X$ and $r>0$. For $f \in L^{p}$ we write $f$ as $f=f \chi_{B}+$ $f \chi_{B^{c}}$, where $\chi_{B}$ is the characteristic function of the ball and $\chi_{B^{c}}$ the characteristic function of the complement of the $d$-ball. Then we have,

$$
\begin{aligned}
I_{\alpha}^{\lambda} f(x) & =\int_{X} \frac{d(x, y)^{\alpha}}{\lambda(x, d(x, y))} f(y) \mathrm{d} \mu(y) \\
& =\int_{B} \frac{d(x, y)^{\alpha}}{\lambda(x, d(x, y))} f(y) \mathrm{d} \mu(y)+\int_{B^{c}} \frac{d(x, y)^{\alpha}}{\lambda(x, d(x, y))} f(y) \mathrm{d} \mu(y) \\
& =I_{1}+I_{2} .
\end{aligned}
$$

We estimate the first integral by splitting the ball $B$ in the concentric annuli $C_{j}=$ $B\left(x, 2^{-j+1} r\right)-B\left(x, 2^{-j} r\right), j=1,2, \ldots$. We obtain

$$
\left|I_{1}\right| \leq \sum_{j=1}^{\infty} \int_{C_{j}}|f(y)| \frac{d(x, y)^{\alpha}}{\lambda(x, d(x, y))} \mathrm{d} \mu(y) .
$$

By our assumption on $\lambda$ we can assure that $\lambda$ is of lower type $\alpha$, and hence by Proposition 2.5, we have

$$
\frac{r^{\alpha}}{\lambda(x, r)} \leq c_{1} \frac{s^{\alpha}}{\lambda(x, s)}, \quad s \leq r \text { and } \forall x \in X
$$


From this we obtain

$$
\begin{aligned}
\left|I_{1}\right| & \leq \sum_{j=1}^{\infty} \int_{B\left(x, 2^{-j+1} r\right)}|f(y)| c_{1} \frac{\left(2^{-j} r\right)^{\alpha}}{\lambda\left(x, 2^{-j} r\right)} \mathrm{d} \mu(y) \\
& =c_{1} r^{\alpha} \sum_{j=1}^{\infty}\left(2^{-j}\right)^{\alpha} \frac{1}{\lambda\left(x, 2^{-j} r\right)} \int_{B\left(x, 2^{-j+1} r\right)}|f(y)| \mathrm{d} \mu(y) \\
& \leq c_{1} r^{\alpha} \sum_{j=1}^{\infty}\left(2^{-j}\right)^{\alpha} \frac{\lambda\left(x, 3 K_{1}\left(2^{-j+1} r\right)\right)}{\lambda\left(x, 2^{-j} r\right)} \Omega(x) \tilde{M} f(x) \\
& \leq c_{1} r^{\alpha} \sum_{j=1}^{\infty}\left(2^{-j}\right)^{\alpha}\left(C_{\lambda}\right)^{\ell} \Omega(x) \tilde{M} f(x) \\
& \leq C_{1} r^{\alpha} \Omega(x) \tilde{M} f(x),
\end{aligned}
$$

where $C_{\lambda}$ denotes the constant in $(2.6), \ell$ is a natural number such that $2^{\ell-1}<$ $6 K_{1} \leq 2^{\ell}$ and $\Omega$ is the maximal function defined by $\Omega(x)=\sup _{R>0} \frac{\mu(B(x, R))}{\lambda(x, R)}$. The constant $C_{1}$ depends only on $K_{1}, C_{\lambda}, c_{1}$ and $\alpha$. Furthermore, as we assumed that the underlying metric space $X$ is bounded, there exists a constant $R_{0}>0$ such that $R_{0}=\operatorname{diam} X$. For this $R_{0}$ there exists $m \in \mathbb{N}_{0}$ such that $2^{m} r<R_{0} \leq$ $2^{m+1} r$. Now, setting $D_{k}=B\left(x, 2^{k+1} r\right)-B\left(x, 2^{k} r\right)$, we decompose $B^{c}(x, r)=$ $\bigcup_{k=0}^{m-1} D_{k} \bigcup\left(B\left(x, R_{0}\right) \backslash B\left(x, 2^{m} r\right)\right)$. We estimate the second integral in a similar way, by considering this decomposition of $B^{c}$.

$$
\begin{aligned}
\left|I_{2}\right| & \leq \sum_{k=1}^{m-1} \int_{D_{k}}|f(y)| \frac{d(x, y)^{\alpha}}{\lambda(x, d(x, y))} \mathrm{d} \mu(y)+\int_{B\left(x, R_{0}\right) \backslash B\left(x, 2^{m} r\right)}|f(y)| \frac{d(x, y)^{\alpha}}{\lambda(x, d(x, y))} \mathrm{d} \mu(y) \\
& \leq \sum_{k=1}^{m-1} \int_{B\left(x, 2^{k+1} r\right)}|f(y)| c_{1} \frac{\left(2^{k} r\right)^{\alpha}}{\lambda\left(x, 2^{k} r\right)} \mathrm{d} \mu(y)+\int_{B\left(x, R_{0}\right)}|f(y)| c_{1} \frac{\left(2^{m} r\right)^{\alpha}}{\lambda\left(x, 2^{m} r\right)} \mathrm{d} \mu(y) .
\end{aligned}
$$

Then, we apply Hölder's inequality in each term of the sum to obtain

$$
\begin{aligned}
\left|I_{2}\right| \leq & c_{1} r^{\alpha}\left[\sum_{k=1}^{m-1}\left(\int_{B\left(x, 2^{k+1} r\right)}|f(y)|^{p} \mathrm{~d} \mu(y)\right)^{1 / p}\left(\int_{B\left(x, 2^{k+1} r\right)} \frac{\left(2^{k} r\right)^{\alpha p^{\prime}}}{\left(\lambda\left(x, 2^{k} r\right)\right)^{p^{\prime}}} \mathrm{d} \mu(y)\right)^{1 / p^{\prime}}\right. \\
& \left.+\left(\int_{B\left(x, R_{0}\right)}|f(y)|^{p} \mathrm{~d} \mu(y)\right)^{1 / p}\left(\int_{B\left(x, R_{0}\right)} \frac{\left(2^{m} r\right)^{\alpha p^{\prime}}}{\lambda\left(x, 2^{m} r\right)^{p^{\prime}}} \mathrm{d} \mu(y)\right)^{1 / p^{\prime}}\right]
\end{aligned}
$$


Now, from the hypothesis on the of lower type of $\lambda(x, r)$, the doubling property of $\lambda$ and the definition of the maximal function $\Omega$, we have

$$
\begin{aligned}
\left|I_{2}\right| & \leq c_{1} r^{\alpha}\|f\|_{p}\left[\sum_{k=1}^{m-1} \frac{2^{k \alpha}}{\lambda\left(x, 2^{k} r\right)}\left(\mu\left(B\left(x, 2^{k+1} r\right)\right)\right)^{1 / p^{\prime}}+\frac{\left(2^{m}\right)^{\alpha}}{\lambda\left(x, 2^{m} r\right)}\left(\mu\left(B\left(x, R_{0}\right)\right)\right)^{1 / p^{\prime}}\right] \\
& \leq c_{1} r^{\alpha}\|f\|_{p}\left[\sum_{k=1}^{m-1} C_{\lambda} \frac{2^{k \alpha}}{\lambda\left(x, 2^{k+1} r\right)}\left(\lambda\left(x, 2^{k+1} r\right)\right)^{1 / p^{\prime}}+C_{\lambda} \frac{2^{m \alpha}}{\lambda\left(x, R_{0}\right)}\left(\lambda\left(x, R_{0}\right)\right)^{1 / p^{\prime}}\right] \\
& \leq c_{1} C_{\lambda} r^{\alpha}\|f\|_{p}\left[\sum_{k=1}^{m-1} 2^{k \alpha}\left(\lambda\left(x, 2^{k+1} r\right)\right)^{-1 / p}+2^{m \alpha}\left(\lambda\left(x, R_{0}\right)\right)^{-1 / p}\right] .
\end{aligned}
$$

Since the application $r \rightarrow \lambda(x, r)$ is non-decreasing, we observe that $\lambda(x, r) \leq$ $\lambda\left(x, 2^{k+1} r\right) \leq \lambda\left(x, R_{0}\right), k=0,1, \ldots, m-1$, and we may conclude that

$$
\begin{aligned}
\left|I_{2}\right| & \leq c_{1} C_{\lambda} r^{\alpha}\|f\|_{p}(\lambda(x, r))^{-1 / p}\left[\sum_{j=1}^{m-1} 2^{k \alpha}+2^{m \alpha}\right] \\
& \leq C_{2} r^{\alpha}\|f\|_{p}(\lambda(x, r))^{-1 / p}
\end{aligned}
$$

where $C_{2}$ is a constant that depends only on $c_{1}, C_{\lambda}$ and $\alpha$.

The above estimates for $I_{1}$ and $I_{2}$ imply the following pointwise inequality:

$$
\left|I_{\alpha}^{\lambda} f(x)\right| \leq C_{3}\left(r^{\alpha} \Omega(x) \tilde{M} f(x)+r^{\alpha}\|f\|_{p}(\lambda(x, r))^{-1 / p},\right.
$$

for arbitrary $x \in X$ and $r>0$. Taking into account condition 2.6 we deduce that $\Omega(x) \leq 1$ for all $x \in X$. Hence

$$
\left|I_{\alpha}^{\lambda} f(x)\right| \leq C_{3}\left(r^{\alpha} \tilde{M} f(x)+r^{\alpha}\|f\|_{p}(\lambda(x, r))^{-1 / p}\right),
$$

for arbitrary $x \in X$ and $r>0$. Suppose we can choose $r>0$ such that $\lambda(x, r)=$ $\frac{\|f\|_{p}^{p}}{\tilde{M} f(x)^{p}}$. Then, in that case from the assumption $r^{\alpha} \leq \lambda(x, r)^{\frac{1}{p}-\frac{1}{q(x)}}$ we get

$$
r^{\alpha} \leq\left(\frac{\tilde{M} f(x)}{\|f\|_{p}}\right)^{\frac{p}{q(x)}-1}
$$

Consequently,

$$
\left.\left|I_{\alpha}^{\lambda} f(x)\right| \leq C_{4}(\tilde{M} f(x))^{\frac{p}{q(x)}}\|f\|_{p}^{1-\frac{p}{q(x)}}\right) .
$$

Such choice of $r$ is possible as long as $\lambda(x, r)$ does not exceed $\lambda\left(x, R_{0}\right)$. However, if it does, it is because $\tilde{M} f(x)^{p} \leq\|f\|_{p}^{p} \lambda\left(x, R_{0}\right)^{-1}$ for these $x$ 's and by setting $r=R_{0}=\operatorname{diam}(X)$ in 4.1 , we have

$$
\left|I_{\alpha}^{\lambda} f(x)\right| \leq C_{5}\|f\|_{p}
$$

Adding 4.2 and (4.3) we obtain that for all $x$ in $X$,

$$
\left|I_{\alpha}^{\lambda} f(x)\right| \leq C_{6}\|f\|_{p}\left(\tilde{M} f(x)^{\frac{p}{q(x)}}\|f\|_{p}^{-\frac{p}{q(x)}}+1\right) .
$$


By 4.4 and Lemma 2.4 it readily follows that

$$
\left\|I_{\alpha}^{\lambda} f\right\|_{q(\cdot)} \leq c\|f\|_{p} .
$$

Indeed, since $I_{p(\cdot)}$ is order preserving and a convex modular (see Section 2 we get

$$
\begin{aligned}
\int_{X}( & \left.\frac{\left|I_{\alpha}^{\lambda} f(x)\right|}{C_{6} 2\left(C_{0}^{p}+\mu(X)\right)^{\frac{1}{q(x)}}\|f\|_{p}}\right)^{q(x)} \mathrm{d} \mu(x) \\
& \leq \int_{X}\left(\frac{\tilde{M} f(x)^{\frac{p}{q(x)}}\|f\|_{p}^{-\frac{p}{q(x)}}+1}{2\left(C_{0}^{p}+\mu(X)\right)^{\frac{1}{q(x)}}}\right)^{q(x)} \mathrm{d} \mu(x) \\
& \leq \int_{X}\left(\frac{1}{2} \frac{\tilde{M} f(x)^{\frac{p}{q(x)}}\|f\|_{p}^{-\frac{p}{q(x)}}}{\left(C_{0}^{p}+\mu(X)\right)^{\frac{1}{q(x)}}}+\frac{1}{2} \frac{1}{\left(C_{0}^{p}+\mu(X)\right)^{\frac{1}{q(x)}}}\right)^{q(x)} \mathrm{d} \mu(x) \\
& \leq \frac{1}{2} \frac{1}{C_{0}^{p}+\mu(X)}\left(\int_{X} \frac{|\tilde{M} f(x)|^{p}}{\|f\|_{p}^{p}} \mathrm{~d} \mu(x)+\int_{X} 1 \mathrm{~d} \mu(x)\right) \\
& =\frac{1}{C_{0}^{p}+\mu(X)}\left(\frac{\|\tilde{M} f\|_{p}^{p}}{\|f\|_{p}^{p}}+\mu(X)\right) \\
& \leq \frac{1}{C_{0}^{p}+\mu(X)}\left(C_{0}^{p} \frac{\|f\|_{p}^{p}}{\|f\|_{p}^{p}}+\mu(X)\right) \\
& =1
\end{aligned}
$$

where $C_{0}$ is the constant in the inequality $\|\tilde{M} f\|_{p} \leq C_{0}\|f\|_{p}$ from Lemma 2.4 .

Now

$$
\begin{aligned}
\left\|I_{\alpha}^{\lambda} f\right\|_{q(\cdot)} & \leq C_{6} 2\left(C_{0}^{p}+\mu(X)\right)^{\frac{1}{q(x)}}\|f\|_{p} \\
& \leq C_{6} 2 \max \left\{\left(C_{0}^{p}+\mu(X)\right)^{\frac{1}{q_{+}}},\left(C_{0}^{p}+\mu(X)\right)^{\frac{1}{q_{-}}}\right\}\|f\|_{p} \\
& =c\|f\|_{p},
\end{aligned}
$$

where we used that if $\left(C_{0}^{p}+\mu(X)\right) \geq 1$ then $\left(C_{0}^{p}+\mu(X)\right)^{\frac{1}{q(x)}} \leq\left(C_{0}^{p}+\mu(X)\right)^{\frac{1}{q_{-}}}$ since $\frac{1}{q(x)} \leq \frac{1}{q_{-}}$, and if $\left(C_{0}^{p}+\mu(X)\right)<1$ then $\left(C_{0}^{p}+\mu(X)\right)^{\frac{1}{q(x)}} \leq\left(C_{0}^{p}+\mu(X)\right)^{\frac{1}{q_{+}}}$ since $\frac{1}{q(x)} \geq \frac{1}{q_{+}}$.

Remark 4.2. In the proof above we have used the maximal function

$$
\Omega(x)=\sup _{R>0} \frac{\mu(B(x, R))}{\lambda(x, R)}
$$

which describes a geometric property of the measure of the $d$-balls. This idea was taken from [9], where it was used for the case $\lambda(x, r)=C r^{n}$.

If $\mu$ is assumed upper Ahlfors $n(\cdot)$-regular, we obtain as a corollary boundedness properties for $I_{\alpha}^{n(\cdot)}$, since $\lambda(x, r)=r^{n(x)}$ for all $x \in X$ and $r>0$ and provided that $0<\alpha<\inf _{X} n(x)$. 
Corollary 4.3. Let us assume that $(X, d)$ is a bounded geometrically doubling quasi-metric space and $\mu$ is a Borel measure on $X$ defined on d-balls, which is finite on bounded sets and positive on d-balls such that $\mu(B(x, r)) \leq r^{n(x)}$ for all $x \in X, 0<r \leq \operatorname{diam}(X)$ and $\inf _{X} n(x)>0$. Let $0<\alpha<\inf _{X} n(x)$. Let $q$ be $a$ measurable function defined on $X$, such that $1<p<q_{-} \leq q(x) \leq q_{+}<\infty$ and $q(x)$ satisfies $\frac{1}{q(x)}=\frac{1}{p}-\frac{\alpha}{n(x)}$ for all $x \in X$. Then

$$
I_{\alpha}^{n(\cdot)}: L^{p}(X, d, \mu) \rightarrow L^{q(\cdot)}(X, d, \mu)
$$

boundedly.

If $f \in L^{p(\cdot)}$ and $p$ is a log-Hölder continuous exponent, i.e.,

$$
|p(x)-p(y)| \leq \frac{C}{-\log |x-y|}, \quad x, y \in X,|x-y|<\frac{1}{2},
$$

Diening [8] has shown that this condition is sufficient on bounded domains for the boundedness on $L^{p(\cdot)}$ of the non-centered maximal operator defined by

$$
\bar{M} f(x):=\sup _{B \ni x} \frac{1}{\mu(B)} \int_{B}|f| \mathrm{d} \mu .
$$

Since $\tilde{M} f$ defined in $(2.8)$ is less than or equal to $\bar{M} f$, the proof of the above theorem can be slightly modified to obtain the boundedness of $I_{\alpha}^{\lambda}$ in the setting of variable exponent Lebesgue spaces. More precisely,

Theorem 4.4. Let us assume that $(X, d)$ is a bounded geometrically doubling quasimetric space and $\mu$ is a Borel measure on $X$ defined on d-balls which is finite on bounded sets and positive on d-balls. Let $\lambda: X \times \mathbb{R}^{+} \rightarrow \mathbb{R}^{+}$be a function, which as a function of the variable $r$ and uniformly on $x$ is non-decreasing, doubling, of lower type greater than $\alpha$ with $\alpha>0$. Let $p$ and $q$ be measurable functions defined on $X$, such that $1<p_{-} \leq p(x)<q(x) \leq q_{+}<\infty$, $p(x)$ satisfies 4.5 and $q(x)$ satisfies $r^{\alpha} \leq \lambda(x, r)^{\frac{1}{p(x)}-\frac{1}{q(x)}}$ for all $x \in X$ and $r>0$. Then

$$
I_{\alpha}^{\lambda} f: L^{p(\cdot)}(X, d, \mu) \rightarrow L^{q(\cdot)}(X, d, \mu)
$$

boundedly if

$$
\mu(B(x, r)) \leq \lambda(x, r)
$$

for all $x \in X$ and $0<r \leq \operatorname{diam}(X)$.

In the next lemma we prove an estimate from below for the norm of the characteristic function of a $d$-ball with measure less than or equal to one. In [6], Capone, Cruz Uribe and Fiorenza give an estimate from above for the norm of the characteristic function of a $d$-ball with measure less than or equal to one.

Lemma 4.5. Let $(X, d, \mu)$ be a quasi-metric measure space, $\mu$ a finite measure over d-balls. Let $q$ be a measurable function defined on $X$ such that $1<q_{-} \leq$ $q(x) \leq q_{+}<\infty$ for every $x \in X$. Then for any $d$-ball $B$ such that $\mu(B) \leq 1$,

$$
\left\|\chi_{B}\right\|_{p(\cdot)} \geq C \mu(B)^{1 / p(x)},
$$

for every $x \in B$. 
Proof. Since $\mu(B) \leq 1$, it is always true for $\lambda \geq 1$ that $\int_{B} \lambda^{-p(x)} \mathrm{d} \mu(x) \leq \mu(B) \leq 1$, and since $p^{+}<\infty$, by the definition of the norm on $L^{p(\cdot)}(X)$ we get

$$
\begin{aligned}
\left\|\chi_{B}\right\|_{p(\cdot)} & =\inf \left\{\lambda>0: \int_{B} \lambda^{-p(x)} \mathrm{d} \mu(x) \leq 1\right\} \\
& =\inf \left\{0<\lambda<1: \int_{B} \lambda^{-p(x)} \mathrm{d} \mu(x) \leq 1\right\} \\
& \geq \inf \left\{0<\lambda<1: \int_{B} \lambda^{-p_{+}(B)} \mathrm{d} \mu(x) \leq 1\right\} \\
& =\mu(B)^{1 / p_{+}(B)}
\end{aligned}
$$

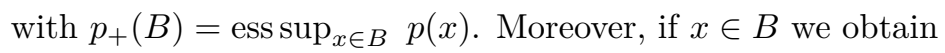

$$
\begin{aligned}
\mu(B)^{1 / p_{+}(B)} & =\mu(B)^{1 / p(x)} \mu(B)^{1 / p_{+}(B)-1 / p(x)} \\
& \geq \mu(B)^{1 / p(x)} \mu(B)^{\frac{p(x)-p_{+}(B)}{p_{+}(B) p_{-}(B)}} \\
& \geq \mu(B)^{1 / p(x)} \mu(B)^{\frac{p_{+}(B)-p_{+}(B)}{p_{+}(B) p_{-}(B)}} \\
& =\mu(B)^{1 / p(x)} .
\end{aligned}
$$

Now we state the second half of our main result.

Theorem 4.6 (Necessary Condition). Let us assume that $(X, d)$ is a bounded geometrically doubling quasi-metric space and $\mu$ is a Borel measure on $X$ defined on $d$-balls which is finite on bounded sets and positive on d-balls. Let $\lambda: X \times \mathbb{R}^{+} \rightarrow \mathbb{R}^{+}$ be a function, which as a function of the variable $r$ and uniformly on $x$ is nondecreasing, doubling, of lower type greater than $\alpha$ with $\alpha>0$. Let $q$ be a measurable function defined on $X$, such that $1<p<q_{-} \leq q(x) \leq q_{+}<\infty$ and $q(x)$ satisfies $r^{\alpha}=\lambda(x, r)^{\frac{1}{p}-\frac{1}{q(x)}}$ for all $x \in X$ and $r>0$. If

$$
I_{\alpha}^{\lambda}: L^{p}(X, d, \mu) \rightarrow L^{q(\cdot)}(X, d, \mu)
$$

boundedly then

$$
\mu(B(x, r)) \leq \lambda(x, r)
$$

for all $x \in X$ and $0<r \leq \operatorname{diam}(X)$.

Proof. Let $I_{\alpha}^{\lambda}$ be bounded from $L^{p}$ into $L^{q(\cdot)}$ and set $f=\chi_{B(a, r)} \frac{\lambda(\cdot, r)}{\lambda(a, r)}$, with $a \in X$ and $r>0$. First we estimate

$$
\int_{X}\left(\chi_{B(a, r)}(x) \frac{\lambda(x, r)}{\lambda(a, r)}\right)^{p} \mathrm{~d} \mu(x)=\int_{B(a, r)}\left(\frac{\lambda(x, r)}{\lambda(a, r)}\right)^{p} \mathrm{~d} \mu(x) \leq \mu(B(a, r)),
$$

since $d(x, a)<r$ implies $\lambda(x, r) \leq \lambda(a, r)$ according to 2.7). Then $\left\|I_{\alpha}^{\lambda} f\right\|_{q(\cdot)} \leq$ $C \mu(B(a, r))^{\frac{1}{p}}$. It readily follows from 2.10 that

$$
\int_{X}\left|\frac{I_{\alpha}^{\lambda}\left(\chi_{B(a, r)}(x) \frac{\lambda(x, r)}{\lambda(a, r)}\right)}{C \mu(B(a, r))^{\frac{1}{p}}}\right|^{q(x)} \mathrm{d} \mu(x) \leq 1 .
$$


For each $x \in B(a, r)$, recalling 2.11, we have

$$
\begin{aligned}
\frac{I_{\alpha}^{\lambda}\left(\chi_{B(a, r)}(x) \frac{\lambda(x, r)}{\lambda(a, r)}\right)}{C \mu(B(a, r))^{\frac{1}{p}}} & =\frac{1}{C \mu(B(a, r))^{\frac{1}{p}}} \int_{X} \chi_{B(a, r)}(x) \frac{\lambda(x, r)}{\lambda(a, r)} \frac{d(x, y)^{\alpha}}{\lambda(x, d(x, y))} \mathrm{d} \mu(y) \\
& \geq \frac{1}{C c_{1} \mu(B(a, r))^{\frac{1}{p}}} \int_{B(a, r)} \frac{\lambda(x, r)}{\lambda(a, r)} \frac{\left(2 K_{1} r\right)^{\alpha}}{\lambda\left(x, 2 K_{1} r\right)} \mathrm{d} \mu(y) \\
& =\frac{\left(2 K_{1}\right)^{\alpha}}{C c_{1}\left(C_{\lambda}\right)^{\ell}} \frac{r^{\alpha}}{\lambda(a, r)} \mu(B(a, r))^{1-\frac{1}{p}}
\end{aligned}
$$

Therefore

$$
\begin{aligned}
\int_{B(a, r)}\left|\frac{\left(2 K_{1}\right)^{\alpha}}{C c_{1}\left(C_{\lambda}\right)^{\ell}} \frac{r^{\alpha}}{\lambda(a, r)} \mu(B(a, r))^{1-\frac{1}{p}}\right|^{q(x)} \mathrm{d} \mu(x) \\
\leq \int_{X}\left|\frac{I_{\alpha}^{\mu_{\lambda}}\left(\chi_{B(a, r)}(x) \frac{\lambda(x, r)}{\lambda(a, r)}\right)}{C \mu(B(a, r))^{\frac{1}{p}}}\right|^{q(x)} \mathrm{d} \mu(x) \leq 1 .
\end{aligned}
$$

Consequently, by the definition of the norm given in 2.9 we get

$$
\frac{\left(2 K_{1}\right)^{\alpha}}{C c_{1}\left(C_{\lambda}\right)^{\ell}} \frac{r^{\alpha}}{\lambda(a, r)} \mu(B(a, r))^{1-\frac{1}{p}}\left\|_{B(a, r)}\right\|_{q(\cdot)} \leq 1 .
$$

As noted in Subsection 2.3 we have $L^{q(\cdot)} \subseteq L^{q_{-}}$since $q_{-} \leq q(x)$ for all $x \in X$, then

$$
\begin{aligned}
\frac{\left(2 K_{1}\right)^{\alpha}}{C c_{1}\left(C_{\lambda}\right)^{\ell}} \frac{r^{\alpha}}{\lambda(a, r)} \mu(B(a, r))^{1-\frac{1}{p}} & \left\|\chi_{B(a, r)}\right\|_{q_{-}} \\
& \leq \frac{\left(2 K_{1}\right)^{\alpha}}{C c_{1}\left(C_{\lambda}\right)^{\ell}} \frac{r^{\alpha}}{\lambda(a, r)} \mu(B(a, r))^{1-\frac{1}{p}}\left\|\chi_{B(a, r)}\right\|_{q(\cdot)} .
\end{aligned}
$$

Now, if $\mu(B(a, r)) \geq 1$ we have

$$
\frac{\left(2 K_{1}\right)^{\alpha}}{C c_{1}\left(C_{\lambda}\right)^{\ell}} \frac{r^{\alpha}}{\lambda(a, r)} \mu(B(a, r))^{1-\frac{1}{p}+\frac{1}{q(a)}} \leq \frac{1}{\tilde{C}} \frac{r^{\alpha}}{\lambda(a, r)} \mu(B(a, r))^{1-\frac{1}{p}+\frac{1}{q_{-}}} \leq 1 .
$$

If instead $\mu(B(a, r)) \leq 1$, we use in 4.7 the estimate of $\left\|\chi_{B(a, r)}\right\|_{q(\cdot)}$ given in Lemma 4.5 and then we also obtain

$$
\frac{\left(2 K_{1}\right)^{\alpha}}{C\left(C_{\lambda}\right)^{\ell}} \frac{r^{\alpha}}{\lambda(a, r)} \mu(B(a, r))^{1-\frac{1}{p}+\frac{1}{q(a)}} \leq 1 .
$$

Using that $r^{\alpha}=\lambda(x, r)^{\frac{1}{p}-\frac{1}{q(x)}}$ for all $x \in X$ we get

$$
\mu(B(a, r))^{1-\frac{1}{p}+\frac{1}{q(a)}} \leq \tilde{C} \frac{\lambda(a, r)}{r^{\alpha}}=\tilde{C} \lambda(a, r)^{1-\frac{1}{p}+\frac{1}{q(a)}} .
$$

From the last inequality we conclude that $\mu(B(x, r)) \leq C^{\prime} \lambda(x, r)$ holds and thus the proof is complete.

Remark 4.7. Notice that the proof of Theorem 1.2 follows immediately from Theorem 4.1 and Theorem 4.6 . It is worth mentioning that in proving the sufficient 
condition we only used there the inequality $r^{\alpha} \leq \lambda(x, r)^{\frac{1}{p}-\frac{1}{q(x)}}$ for all $x \in X$ and $r>0$.

Remark 4.8. When $\lambda(x, r)=r^{n}$ the condition about the upper type of $\lambda$ implies that $0<\alpha<n$ and the assumption $r^{\alpha}=\lambda(x, r)^{\frac{1}{p}-\frac{1}{q(x)}}$ takes the form $\frac{1}{q}=\frac{1}{p}-\frac{\alpha}{n}$, because from $r^{\alpha}=\left(r^{n}\right)^{\frac{1}{p}-\frac{1}{q(x)}}$ we get that $q$ is constant and satisfies the equality mentioned above.

Corollary 4.9. Let us assume that $(X, d)$ is a bounded geometrically doubling quasi-metric space and $\mu$ is a Borel measure on $X$ defined on d-balls which is finite on bounded sets and positive on d-balls. Let $q$ be a measurable function defined on $X$. If $1<p<q_{-} \leq q(x) \leq q_{+}<\infty$ and $q(x)$ satisfies $\frac{1}{q(x)}=\frac{1}{p}-\frac{\alpha}{n(x)}$ with $0<\alpha<\inf _{X} n(x)$, then the condition $\mu(B(x, r)) \leq \tilde{C} r^{n(x)}$ for some constant $\tilde{C}$ is necessary for $\left\|I_{\alpha}^{n(\cdot)} f\right\|_{q(\cdot)} \leq C\|f\|_{p}$ to hold.

\section{RiEsz type Potential in AN ENVIRONMENT DOUbling With two COMPONENTS OF DIFFERENT DIMENSIONS}

5.1. More examples of upper doubling measure. One of the authors and Aimar considered in $[3$ the problem of defining a measure when the metric measure space $X$ is formed by two sets $X_{1}$ and $X_{2}$ of different dimensions under certain conditions on contact. It is easy to obtain the same result considering quasi-metric spaces instead of metric spaces. If each component $X_{i}$ supports a measure $\mu_{i}$, $i=1,2$, we can add these measures and obtain a measure supported on the whole space. However $\mu_{1}+\mu_{2}$ is not necessarily doubling on $X$. In [3] these natural measures $\mu_{i}, i=1,2$, are modified by introducing some weights depending on the distance to the contact point. This is done in order to get a doubling measure for the whole space. In this section we will show that the measure defined in [3] gives a non-trivial example of upper doubling measure, since it is not only doubling but also variable upper Ahlfors regular. Our setting is characterized by defining the following three elements:

[a] The pieces of $X$.

$X=X_{1} \cup X_{2} \cup\left\{x_{0}\right\}$ and $(X, d)$ is a bounded metric space.

[b] Contact of order zero.

The components of $X$ have contact of order zero in $x_{0}$ or $X$ satisfies the property $\mathcal{C}_{o}$ if and only if $\left\{x_{0}\right\}=\bar{X}_{1} \cap \bar{X}_{2}$ and $d\left(x, x_{0}\right) \leq c\left[d\left(x, X_{1}\right)+d\left(x, X_{2}\right)\right]$ for some constant $c$ and every $x \in X$.

[c] Dimensions.

$\left(X_{i}, d, \mu_{i}\right)$ is an Ahlfors $n_{i}$-regular metric measure space with $0<n_{1} \leq n_{2}<\infty$.

Let us observe that since $x_{0} \in \overline{X_{1}} \cap \overline{X_{2}}$ we have the inequality $d\left(x, x_{0}\right) \geq d\left(x, X_{1}\right)+$ $d\left(x, X_{2}\right)$, for every $x \in X$. Hence if $X$ satisfies $\mathcal{C}_{o}$ the constant $c$ is at least one. On the other hand, property $\mathcal{C}_{o}$ provides a pointwise equivalence of the functions $d\left(x, x_{0}\right)$ and $d\left(x, X_{1}\right)+d\left(x, X_{2}\right)$.

It is easy to see that property $\mathcal{C}_{0}$ is equivalent to the existence of a constant $\bar{c}>0$ such that for every $x \in X_{i}$ it is true that $B\left(x, \bar{c} d\left(x, x_{0}\right)\right) \cap X_{j}=\emptyset, i \neq j$. 
As it is known, fractal sets produced by the Hutchinson iteration scheme ([24]), under the open set condition, are in fact spaces of homogeneous type with the right Hausdorff measure, which are Ahlfors $Q$-regular for some positive real number $Q$. That is the case, for example, of the middle thirds Cantor sets and Sierpinski gaskets.

The context described is a natural abstraction of many situations of fractal fields (see for example [17]) with a special order of contact. For instance, we could take a plate joined to a block, or a rod joined to a plate, or a Cantor set joined to a plate, or a Cantor set joined to a Sierpinski gasket, etc.

In this environment, in [3] some "weights" are introduced to the Ahlfors $n_{i^{-}}$ regular measures, $i=1,2$, in order to get a doubling measure for the whole space $X=\bigcup_{i=1}^{2} X_{i} \cup\left\{x_{0}\right\}$. More precisely, they prove the following theorem.

Theorem 5.1 ([3, Theorem 1.2]). Assume that $X=X_{1} \cup X_{2} \cup\left\{x_{0}\right\}$ satisfies $\mathcal{C}_{0}$. For $i=1,2$ let $\left(X_{i}, d, \mu_{i}\right)$ be a $n_{i}$-normal space with $0<n_{1} \leq n_{2}<\infty$. For $\gamma_{1}>-n_{1}$ and $\gamma_{2}>-n_{2}$, let $\mu^{\gamma_{1}, \gamma_{2}}$ be the measure defined by

$$
\mu^{\gamma_{1}, \gamma_{2}}(E)=\int_{E \cap X_{1}} d\left(x, x_{0}\right)^{\gamma_{1}} \mathrm{~d} \mu_{1}(x)+\int_{E \cap X_{2}} d\left(x, x_{0}\right)^{\gamma_{2}} \mathrm{~d} \mu_{2}(x) .
$$

Then $\left(X, d, \mu^{\gamma_{1}, \gamma_{2}}\right)$ is a space of homogenous type if and only if $\gamma_{1}+n_{1}=\gamma_{2}+n_{2}$.

Let us denote by $\xi$ the number $\gamma_{i}+n_{i}, i=1,2$. So, by the above result, it is enough to require $\xi>0$ to ensure that the pair $\left(\gamma_{1}, \gamma_{2}\right)$ is admissible for the definition of a doubling measure. For our purposes, let $\gamma(x)$ denote the function defined in $X$ by

$$
\gamma(x)= \begin{cases}\gamma_{i}, & \text { if } x \in X_{i}, i=1,2 \\ \gamma_{1}, & \text { if } x=x_{0}\end{cases}
$$

Also we could have chosen $\gamma_{2}$ as image of $x_{0}$. Let $n(x)$ denote the function defined in $X$ by

$$
n(x)= \begin{cases}n_{i}, & \text { if } x \in X_{i}, i=1,2 \\ n_{1}, & \text { if } x=x_{0}\end{cases}
$$

or $n_{2}$ as image of $x_{0}$.

Although the measure $\mu^{\gamma_{1}, \gamma_{2}}$ is not an Ahlfors $Q$-regular measure for some $Q>0$, the following theorem gives us estimates of $\mu^{\gamma_{1}, \gamma_{2}}$ on balls of $(X, d)$ similar to those of the inequalities of Ahlfors $Q$-regular measures.

Theorem 5.2 ([3, Theorem 3.2]). Assume that $\left(X_{1}, X_{2}, d\right)$ satisfies $\mathcal{C}_{0}$. For $i=1,2$ let $\mu_{i}$ be a Borel measure on $\left(X_{i}, d\right)$ such that $\left(X_{i}, d, \mu_{i}\right)$ is a $n_{i}$-normal space, with $0<n_{1} \leq n_{2}<\infty$. For $\gamma_{1}>-n_{1}$ and $\gamma_{2}>-n_{2}$, let $\mu^{\gamma_{1}, \gamma_{2}}$ be the measure defined by (5.1). Assume that $\gamma_{1}-\gamma_{2}=n_{2}-n_{1}$ and set $\xi=\gamma_{1}+n_{1}=\gamma_{2}+n_{2}=\gamma(x)+n(x)$. Then, there exist purely geometric constants $1 \leq K_{3}<\infty$ and $1>c>0$, such that given $x \in X=X_{1} \cup X_{2}$ and $r>0$ we have

(i) $K_{3}^{-1} d\left(x, x_{0}\right)^{\gamma(x)} r^{n(x)} \leq \mu^{\gamma_{1}, \gamma_{2}}(B(x, r)) \leq K_{3} d\left(x, x_{0}\right)^{\gamma(x)} r^{n(x)}$, for $x \in X$ and $r<c d\left(x, x_{0}\right)$; 
(ii) $K_{3}^{-1} r^{\xi} \leq \mu^{\gamma_{1}, \gamma_{2}}(B(x, r)) \leq K_{3} r^{\xi}$, for $c d\left(x, x_{0}\right) \leq r \leq S:=\operatorname{diam}\left(X_{1}\right)+$ $\operatorname{diam}\left(X_{2}\right)$;

(iii) $\mu^{\gamma_{1}, \gamma_{2}}(B(x, r))=\mu^{\gamma, \gamma_{2}}\left(X_{1}\right)+\mu^{\gamma_{1}, \gamma_{2}}\left(X_{2}\right)$, for $r>S$.

We note that in the statement of the above theorem it is possible to consider the contact point $x_{0}$ as an element of $X$. In such case it is easy to obtain, using a density argument, that $\mu^{\gamma_{1}, \gamma_{2}}\left(B\left(x_{0}, r\right)\right) \approx r^{\xi}$ for all $r>0$.

Therefore, the estimates in the above theorem can be summarized as follows:

$$
K_{3}^{-1} r^{\xi} \leq \mu^{\gamma_{1}, \gamma_{2}}(B(x, r)) \leq \begin{cases}K_{3} r^{n(x)} d\left(x, x_{0}\right)^{\gamma(x)} & \text { if } r<c d\left(x, x_{0}\right), \\ K_{3} r^{\xi} & \text { if } r \geq c d\left(x, x_{0}\right),\end{cases}
$$

where $\gamma(x)$ and $n(x)$ denote the functions defined by $(5.2)$ and $(5.3)$ respectively. Thus (5.4) provides a new example of an upper doubling measure, with dominating function defined by

$$
\lambda(x, r)= \begin{cases}K_{3} r^{n(x)} d\left(x, x_{0}\right)^{\gamma(x)} & \text { if } r<c d\left(x, x_{0}\right), \\ K_{3} r^{\xi} & \text { if } r \geq c d\left(x, x_{0}\right)\end{cases}
$$

It is easy to check that the function $\lambda$ satisfies the required properties.

The inequalities (5.4) and (5.6) show that $\mu^{\gamma_{1}, \gamma_{2}}$ is a lower Ahlfors $\xi$-regular measure, which is also a variable upper Ahlfors regular measure, with $\xi \geq n(x)$ for all $x \in X$. As this measure is lower Ahlfors regular, there are no isolated points; and as it is doubling, it is not atomic (see [32]).

On the other hand, using the fact that $(X, d)$ is bounded, we have that $d\left(x, x_{0}\right)$ is less than or equal to $R_{0}=\operatorname{diam} X$. With these observations we can obtain another simpler expression for $\lambda$. In fact,

$$
\mu^{\gamma_{1}, \gamma_{2}}(B(x, r)) \leq \lambda(x, r)=K_{4} r^{n(x)},
$$

where $K_{4}$ is a constant depending of $K_{3}, R_{0}, \gamma_{1}$ and $\gamma_{2}$. It is not difficult to prove that $\lambda(x, r)=K_{4} r^{n(x)}$ as a function of the variable $r$ and uniformly on $x$ is of lower type $n_{1}$. So, by Remark 2.3 and Lemma 2.1 the measure $\mu^{\gamma_{1}, \gamma_{2}}$ is not atomic.

In the setting described by [a], [b] and [c] above and for a function $f$ defined on $X$ by $f=f_{1} \chi_{X_{1}}+f_{2} \chi_{X_{2}}$ belonging to $L_{\text {loc }}^{1}\left(X, d, \mu^{\gamma_{1}, \gamma_{2}}\right)$, the corresponding Hardy-Littlewood maximal function is given by

$$
\begin{aligned}
\mathcal{M} f(x) & =\sup _{x \in B} \frac{1}{\mu^{\gamma_{1}, \gamma_{2}}(B)} \int_{B}|f(y)| \mathrm{d} \mu^{\gamma_{1}, \gamma_{2}}(y) \\
& =\sup _{x \in B} \frac{\int_{B \cap X_{1}}\left|f_{1}(y)\right| d\left(y, x_{0}\right)^{\gamma_{1}} \mathrm{~d} \mu_{1}(y)+\int_{B \cap X_{2}}\left|f_{2}(y)\right| d\left(y, x_{0}\right)^{\gamma_{2}} \mathrm{~d} \mu_{2}(y)}{\int_{B \cap X_{1}} d\left(y, x_{0}\right)^{\gamma_{1}} \mathrm{~d} \mu_{1}(y)+\int_{B \cap X_{2}} d\left(y, x_{0}\right)^{\gamma_{2}} \mathrm{~d} \mu_{2}(y)} .
\end{aligned}
$$

This maximal operator $\mathcal{M}$ is bounded on $L^{p}\left(X, d, \mu^{\gamma_{1}, \gamma_{2}}\right)$ since $\mu^{\gamma_{1}, \gamma_{2}}$ is doubling. Moreover $\mathcal{M}$ is bounded on $L^{p}(w d \mu)$ if and only if $w \in A_{p}\left(X, d, \mu^{\gamma_{1}, \gamma_{2}}\right)$. In [2] necessary and sufficient conditions on two Muckenhoupt $A_{p}$ weights, defined on each of the two components of a space of homogeneous type touching at a single point, are given in order to obtain an $A_{p}$ weight on the whole space. 
5.2. Boundedness of $I_{\alpha}^{n(\cdot)}$ with measure $\mu=\mu^{\gamma_{1}, \gamma_{2}}$. Since for the measure $\mu^{\gamma_{1}, \gamma_{2}}$ the upper dominating function is $K_{4} r^{n(x)}$, we can obtain from 3.2 that the Riesz type potential operator associated with the measure $\mu^{\gamma_{1}, \gamma_{2}}$ is given by

$$
I_{\alpha}^{n(\cdot)} f(x)=\int_{X} \frac{d(x, y)^{\alpha}}{d(x, y)^{n(x)}} f(y) \mathrm{d} \mu^{\gamma_{1}, \gamma_{2}}(y) .
$$

The boundedness of this operator for functions in $L^{p}\left(X, d, \mu^{\gamma_{1}, \gamma_{2}}\right)$ follows as an immediate consequence of Theorem 4.1. More precisely, we have the following result.

Theorem 5.3. Let us assume that $X=X_{1} \cup X_{2} \cup\left\{x_{0}\right\}$ satisfies $\mathcal{C}_{0}$ and $(X, d)$ is a bounded quasi-metric space. Let $\mu_{i}, i=1,2$, be a Borel measure defined on $\left(X_{i}, d\right)$ such that $\left(X_{i}, d, \mu_{i}\right)$ is an Ahlfors $n_{i}$-regular space, with $0<n_{1} \leq n_{2}<\infty$. For $\gamma_{1}>-n_{1}$, and $\gamma_{2}>-n_{2}$, let $\mu^{\gamma_{1}, \gamma_{2}}$ be the measure that satisfies (5.6) with $\gamma_{1}-\gamma_{2}=n_{2}-n_{1}$ and $\gamma_{i}>0$. Let $q$ be a measurable function defined on $X$. If $1<p<q_{-} \leq q(x) \leq q_{+}<\infty$ and $q(x)$ satisfies $\frac{1}{q(x)}=\frac{1}{p}-\frac{\alpha}{n(x)}$, for all $x \in X$, $0<\alpha<n_{1}$ and where $n(x)$ is given by $(5.3)$, then the operator $I_{\alpha}^{n(\cdot)}$ is a bounded operator from $L^{p}\left(X, d, \mu^{\gamma_{1}, \gamma_{2}}\right)$ to $L^{q(\cdot)}\left(X, d, \mu^{\gamma_{1}, \gamma_{2}}\right)$.

Remark 5.4. Note that in the hypotheses of Theorem 5.3 it is not assumed that $(X, d)$ is geometrically doubling as in Theorem 4.1 because, as it was as mentioned in Section 2, if $(X, d)$ supports a doubling measure then $(X, d)$ is geometrically doubling. On the other hand, the condition about lower type of $\lambda$ when $\lambda(x, r)=$ $K_{4} r^{n(x)}$ leads to $\alpha<n_{1}$.

Remark 5.5. The case of the boundedness of $I_{\alpha}^{n(\cdot)}$ for functions in $L^{p}(X, d, \mu)$ under the same assumptions on $(X, d)$ as in Theorem 5.3 , except that the measure is $\mu_{1}+\mu_{2}$, is studied in [28].

\section{REFERENCES}

[1] Aimar, H. Distance and Measure in Analysis and P.D.E., to be published by Birkhäuser.

[2] Aimar, H., Iaffei, B. and Nitti, L. Pasting Muckenhoupt weights through a contact point between sets of different dimensions. Acta Math. Hungar. 129 (2010), 368-377. MR 2739770.

[3] Aimar, H. and Nitti, L. Separation and contact of sets of different dimensions in a doubling environment. Publ. Math. Debrecen 74 (2009), 351-368. MR 2521380

[4] Almeida, A. and Samko, S. Fractional and hypersingular operators in variable exponent spaces on metric measure spaces. Mediterr. J. Math. 6 (2009), 215-232. MR 2516251

[5] Avnir, D., Farin, D. and Pfeifer, P. Molecular fractal surfaces. Nature 308 (1984), 261-263.

[6] Capone, C. and Cruz-Uribe, D. and Fiorenza, A. The fractional maximal operator and fractional integrals on variable $L^{p}$ spaces. Rev. Mat. Iberoam. 23 (2007), 743-770. MR 2414490.

[7] Coifman, R. R. and Weiss, G. Analyse harmonique non-commutative sur certains espaces homogènes, Lecture Notes in Mathematics, vol. 242, Springer-Verlag, Berlin, 1971. MR 0499948.

[8] Diening, L. Maximal function on generalized Lebesgue spaces $L^{p(\cdot)}$. Math. Inequal. Appl. 7 (2004), 245-253. MR 2057643

[9] Edmunds, D. E., Kokilashvili, V. and Meskhi, A. Bounded and compact integral operators, Mathematics and its Applications, 543. Kluwer Academic Publishers, Dordrecht, 2002. MR 1920969

[10] Farin, D., Volpert, A. and Avnir, D. Determination of Adsorption Conformation from Surface Resolution Analysis. J. Am. Chem. 107 (1985), 3368-3370. 
[11] Fu, Xing, Yang, Dachun and Yuan, Wen. Generalized fractional integrals and their commutators over non-homogeneous metric measure spaces, Taiwanese J. Math. 18 (2014), 509-557. MR 3188518

[12] Futamura, T., Mizuta, Y. and Shimomura, T. Sobolev embeddings for variable exponent Riesz potentials on metric spaces. Ann. Acad. Sci. Fenn. Math. 31 (2006), 495-522. MR 2248828

[13] García-Cuerva, J. and Gatto, A. E. Boundedness properties of fractional integral operators associated to non-doubling measures. Studia Math. 162 (2004), 245-261. MR 2047654

[14] Genebashvili, I., Gogatishvili, A., Kokilashvili, V. and Krbec, M. Weight theory for integral transforms on spaces of homogeneous type, Pitman Monographs and Surveys in Pure and Applied Mathematics, 92. Longman, Harlow, 1998. MR 1791462

[15] Gorosito, O., Pradolini, G. and Salinas, O. Boundedness of fractional operators in weighted variable exponent spaces with non doubling measures. Czechoslovak Math. J. 60(135) (2010), 1007-1023. MR 2738962

[16] Hajłasz, P. and Koskela, P. Sobolev met Poincaré. Mem. Amer. Math. Soc. 145 (2000), no. 688. MR 1683160

[17] Hambly, B. M. and Kumagai, T. Diffusion processes on fractal fields: heat kernel estimates and large deviations. Probab. Theory Related Fields 127 (2003), 305-352. MR 2018919

[18] Hardy, G. H. and Littlewood, J. E. Some properties of fractional integrals. I. Math. Z. 27 (1928), 565-606. MR 1544927.

[19] Hardy, G. H. and Littlewood, J. E. Some properties of fractional integrals. II. Math. Z. 34 (1932), 403-439. MR 1545260.

[20] Harjulehto, P., Hästö, P. and Latvala, V. Sobolev embeddings in metric measure spaces with variable dimension. Math. Z. 254 (2006), 591-609. MR 2244368

[21] Harjulehto, P., Hästö, P. and Pere, M. Variable exponent Lebesgue spaces on metric spaces: the Hardy-Littlewood maximal operator. Real Anal. Exchange 30 (2004/05), 87103. MR 2126796

[22] Hedberg, L. I. On certain convolution inequalities. Proc. Amer. Math. Soc. 36 (1972), 505510. MR 0312232

[23] Heinonen, J. Lectures on analysis on metric spaces. Universitext. Springer-Verlag, New York, 2001. MR 1800917

[24] Hutchinson, J. E. Fractals and self-similarity. Indiana Univ. Math. J. 30 (1981), 713-747. MR 0625600

[25] Hytönen, T. A framework for non-homogeneous analysis on metric spaces, and the RBMO space of Tolsa. Publ. Mat. 54 (2010), 485-504. MR 2675934

[26] Hytönen, T. and Martikainen, H. Non-homogeneous Tb theorem and random dyadic cubes on metric measure spaces. J. Geom. Anal. 22 (2012), 1071-1107. MR 2965363

[27] Hytönen, T., Yang, D. and Yang, D. The Hardy space $H^{1}$ on non-homogeneous metric spaces. Math. Proc. Cambridge Philos. Soc. 153 (2012), 9-31. MR 2943664

[28] Iaffei, B. and Nitti, L. Boundedness of generalized Riesz type potentials with variable dimension and doubling radial weights. Preprint.

[29] Kokilashvili, V. and Meskhi, A. Fractional integrals on measure spaces. Fract. Calc. Appl. Anal. 4 (2001), 1-24. MR 1815331

[30] Kováčik, O. and Rákosník, J. On spaces $L^{p(x)}$ and $W^{k, p(x)}$. Czechoslovak Math. J. 41(116) (1991), 592-618. MR 1134951.

[31] Luukkainen, J. and Saksman, E. Every complete doubling metric space carries a doubling measure. Proc. Amer. Math. Soc. 126 (1998), 531-534. MR 1443161

[32] Macías, R. A. and Segovia, C. Lipschitz functions on spaces of homogeneous type. Adv. in Math. 33 (1979), 257-270. MR 0546295

[33] Musielak, J. Orlicz spaces and modular spaces, Lecture Notes in Mathematics, vol. 1034, Springer-Verlag, Berlin, 1983. MR 0724434 
[34] Nazarov, F., Treil, S. and Volberg, A. Weak type estimates and Cotlar inequalities for Calderón-Zygmund operators on nonhomogeneous spaces. Internat. Math. Res. Notices 1998, no. 9, 463-487. MR 1626935

[35] Samko, S. G., Kilbas, A. A. and Marichev, O. I. Fractional integrals and derivatives. Gordon and Breach, Yverdon, 1993. MR 1347689

[36] Sobolev, S. L. On a theorem of functional analysis. Mat. Sb. (N.S.) 4(46) (1938), 471-497, English transl. Amer. Math. Soc. Transl. (2) 34 (1963), 36-68.

[37] Stein, E. M. Singular integrals and differentiability properties of functions. Princeton Mathematical Series, no. 30. Princeton University Press, Princeton, N.J., 1970. MR 0290095

[38] Takayasu, H. Fractals in the physical sciences. Nonlinear Science: Theory and Applications. Manchester University Press, Manchester, 1990. MR 1098503.

[39] Triebel, H. and Yang, D. Spectral theory of Riesz potentials on quasi-metric spaces. Math. Nachr. 238 (2002), 160-184. MR 1900816.

[40] Vol'berg, A. L. and Konyagin, S. V. On measures with the doubling condition. Izv. Akad. Nauk SSSR Ser. Mat. 51 (1987), 666-675; translation in Math. USSR-Izv. 30 (1988), 629638. MR 0903629.

[41] Volberg, A. and Wick, B. D. Bergman-type singular integral operators and the characterization of Carleson measures for Besov-Sobolev spaces and the complex ball. Amer. J. Math. 134 (2012), 949-992. MR 2956255

[42] Wu, J.-M. Hausdorff dimension and doubling measures on metric spaces. Proc. Amer. Math. Soc. 126 (1998), 1453-1459. MR 1443418

[43] Zähle, M. Riesz potentials and Liouville operators on fractals. Potential Anal. 21 (2004), 193-208. MR 2058033

[44] Zähle, M. Harmonic calculus on fractals - a measure geometric approach. II. Trans. Amer. Math. Soc. 357 (2005), 3407-3423. MR 2146630

\author{
Bibiana Iaffei ${ }^{\otimes}$ \\ Departamento de Matemática (FHUC-UNL), IMAL-CONICET, Santa Fe, Argentina \\ biaffei@santafe-conicet.gov.ar
}

Liliana Nitti

Departamento de Matemática (FHUC-UNL), IMAL-CONICET, Santa Fe, Argentina

rnitti@fhuc.unl.edu.ar

Received: October 12, 2016

Accepted: July 10, 2017 